\title{
The Effect of Radial Radiation Transport on Intensity Characteristics and Oscillation Frequency of Homogeneously Broadened Lasers
}

\author{
G. J. ERNST AND W. J. WITTEMAN
}

\begin{abstract}
In the present treatment of performance characteristics of laser systems, having homogeneous line broadening, a radial gain profile is taken into account. It will be shown that such a profile, which may be induced by the laser beam itself, changes the curvature of the phase front, so that additional radiation transport in the radial direction takes place. It turns out that for many practical systems the radial radiation transport can be comparable to the radiation gained directly from the medium and therefore may not be neglected. Furthermore, it will be shown how the additional radial radiation transport affects the oscillating frequency. Conditions are deduced in which mode competition leads to the survival of the mode having highest radiation intensity. This is not the one closest to the line center as found in systems where radial radiation transport is not taken into account. Treating this problem we approximate medium parameters by a quadratic profile so that the modes have a Gaussian structure. The oscillation frequency, determined on one side by the dispersion of the active medium and on the other side by the spontaneous decay of the upper level, is discussed. The frequency shift is not very sensitive to changes of mirror reflectivity but increases considerably with increasing thermal effects. Numerical calculations show, for instance, that for a semiconfocal system a frequency shift close to 0.5 in units of normalized frequency can be predicted.
\end{abstract}

\section{INTRODUCTION}

$\mathbf{C}$ OMPETITION phenomena between oscillating modes of laser systems are well known. Experimental and theoretical studies have been made for systems having either inhomogeneous or homogeneous line broadening [1]-[3]. It is found that as long as the modes have sufficient interaction with each other through the active medium, an initial excitation of all modes above threshold may finally lead to the survival of only the most strongly excited one at the expense of the other modes. The surviving one has the larger gain-over-loss ratio. In general this is, depending on the resonance conditions, a mode with frequency as close as possible to a line center.

Considering the mutual interaction of oscillating modes, the local change of radiation was only described by the local interaction of the field and the medium. If, however, the propagation properties of the interacting wave are also taken into account, the local field strength may also be changed by convergence or divergence of the wavefront. In some laser systems, where the field distribution is such that the two running waves have opposite curvatures at any place, the contributions of the two running waves to the local intensity, as will be shown in the present analysis, just cancel. This means that for such systems mode competition can be described by taking into account the interaction of radiation and matter only.

Manuscript received June 12, 1973; revised August 31, 1973.

The authors are with the Department of Physics, Twente University of Technology, Enschede, The Netherlands.
For systems where a gain profile influences the field distribution, which is practically always the case, the two running waves do not have opposite curvatures. This means that apart from the interaction with the medium, the intensity can also be changed by a curvature effect of the two wavefronts.

The effect of the propagation properties on the local field changes can be considerable and for many laser systems this may not be neglected in the calculations of intensity characteristics and competition phenomena.

In this paper we shall treat this curvature effect for Gaussian beams and a homogeneously broadened medium as present, for instance, in efficient molecular systems ( $\mathrm{CO}$ and $\mathrm{CO}_{2}$ lasers). From experiments it is well known that the gain and, more important, the saturation parameter for a Gaussian beam propagating through a homogeneously broadened medium can be measured [4]; i.e., the Gaussian nature is conserved to a good approximation. Also an efficient high-power laser system with Gaussian output beam has been observed [5]. Previously [6], we reported that for a $\mathrm{CO}_{2}$ laser system with an active medium of $380-\mathrm{cm}$ length the output beam remains Gaussian up to discharge currents of about 12 $\mathrm{mA}$.

These observations support the assumption that the medium parameters can be approximated with a quadratic profile for which it has been shown that a Gaussian beam remains Gaussian while propagating through it. Although in practice no profile is quadratic, it should be noted that for media having axial symmetry a serial expansion of propagation constants in terms of radial distance from the axis shows that the main term near the axis (where the field is strongest) is quadratic. We shall derive these main terms for the gain and dispersion profile and in this way indicate how the predictions can be applied to actual systems.

The curvature effect also has a large effect on the competition phenomena between oscillating modes. It will be shown that the surviving mode is not necessarily the one having the largest gain-over-loss ratio. The most favorable mode to oscillate will have an increasing frequency shift from the line center with increasing pump power and/or decreasing outcoupling. The explanation of this frequency shift is based on the presence of a radial gain profile. Due to dispersion the gain profile is accompanied with an index profile, which in turn has an effect on the curvature of the wavefront. The radiation increase by this curvature effect increases with the frequency shift.

It will be shown that in general this frequency shift is 
further increased if an index profile, for instance, caused by thermal effects of a gas discharge, is present.

We wish to emphasize that in the present analysis some approximations are introduced, i.e., medium parameters are described by a quadratic profile, the medium is homogeneously broadened in which hole burning and coherence effects are neglected, and the sum of the intensities of the running waves is approximated as constant: These approximations are only made for mathematical reasons and for the clarity of presentation. They have no bearing at all on the physical effect of the curvature of the wavefront.

\section{The Differential EQuation fOr a Gaussian Mode in a Lenslike Active Medium}

Kogelnik [7] has derived that a medium with a quadratic radial profile for the complex propagation constant can support Gaussian beams. For such beams he has shown that the beamwidth or spot size $\omega$, which measures the distance at which the field amplitude is $1 / e$ times that on the axis, and the radius of curvature are closely related and can conveniently be combined to a complex-valued beam parameter $q$, defined by

$$
\frac{1}{q(z)}=\frac{1}{R(z)}-j \frac{\lambda}{\pi \omega^{2}(z)}
$$

when $z$ is the direction of propagation and $\lambda$ and $R$ are the wavelength and the radius of curvature of the beam in the medium. $R(z)$ is defined positive for a diverging beam traveling in the positive $z$ direction.

In [6], where the field distributions of Gaussian modes in a lenslike medium were calculated, we conveniently used the dimensionless parameters $P(z), U(z)$, and $Q(z)$, defined by

$$
P(z)=\frac{L}{q(z)}, \quad U(z)=\frac{L}{R(z)}, \quad Q(z)=\frac{\lambda L}{\pi \omega^{2}(z)}
$$

in which $L$ is the length of the tube.

To determine the parameters of the Gaussian beam one can write for the electric field strength in the laser:

$$
E(r, z)=\exp (-j \Phi) \cdot \exp -j\left(T+\frac{1}{2} \frac{r^{2} k_{0}}{q}\right)
$$

in which the time-dependent part has been dropped, and where $T$ is a complex phase parameter, $q$ is the complex beam parameter defined in (1), $\Phi^{\prime}=k_{0}(z)$ is the complex propagation constant at the axis of the tube, and the prime indicates differentiation with respect to $z$.

Substitution of (3) into the scalar wave equation

$$
\Delta E+k^{2} E=0
$$

with

$$
k^{2}(r, z)=k_{0}^{2}(z)-k_{0}(z) \cdot k_{2}(z) r^{2}
$$

gives, after comparison of terms of equal powers of $r$, the following two differential equations:

$$
\begin{gathered}
T^{\prime}=-j\left(\frac{1}{q}+\frac{k_{0}{ }^{\prime}}{2 k_{0}}\right) \\
\frac{1}{q^{2}}+\left(\frac{1}{q}\right)^{\prime}+\frac{k_{0}{ }^{\prime}}{k_{0}} \frac{1}{q}+\frac{k_{2}}{k_{0}}=0
\end{gathered}
$$

When $k_{2}<<k_{0}$, as is the case for visible and infrared light, (5) can be approximated as

$$
k=k_{0}-\frac{1}{2} k_{2} r^{2}
$$

with

$$
\begin{aligned}
& k_{0}=\beta_{0}+j \alpha_{0} \\
& k_{2}=-\beta_{2}-j \alpha_{2} .
\end{aligned}
$$

Written in dimensionless form, (6) and (7) become

$$
\begin{gathered}
\frac{d T}{d \zeta}=-j\left(P+\frac{1}{2 k_{0}} \frac{d k_{0}}{d \zeta}\right) \\
P^{2}+\frac{d P}{d \zeta}+\frac{P}{k_{0}} \frac{d k_{0}}{d \zeta}+\eta^{2}=0
\end{gathered}
$$

where

$$
\begin{aligned}
\zeta & =\frac{z}{L} \\
\eta^{2} & =-(\beta+j \alpha) \\
\beta & =\frac{\beta_{2} L^{2}}{\dot{k}_{0}} \\
\alpha & =\frac{\alpha_{2} L^{2}}{k_{0}} .
\end{aligned}
$$

When $k_{0}$ and $k_{2}$ are independent of $z,(7 \mathrm{a})$ is equivalent to the $A B C D$ law for Gaussian beams [7]. In a previous publication [6] we calculated the influence of the radial index and gain parameters $\beta$ and $\alpha$ on the beam parameters $Q$ and $U$ by applying the $A B C D$ law to a complete round trip through a laser resonator consisting of two mirrors with radii of curvature $R_{1}$ and $R_{2}$, respectively. In the present paper we shall be concerned with (6a) and we shall show how, for homogeneous broadening, competition between different Gaussian modes can force the laser to operate at a frequency different from the central frequency of the line. We shall assume again that $k_{0}$ and $k_{2}$ are not $z$ dependent. By differentiation of (3) for $r=0$ with respect to $\zeta$ it follows:

$$
\frac{1}{E} \frac{d E(\zeta)}{d \zeta}=-j k_{0} L-j \frac{d T}{d \zeta}
$$

Substitution of (6a) into (14) gives:

$$
\frac{1}{E} \frac{d E}{d \zeta}=-j k_{0} L-P
$$




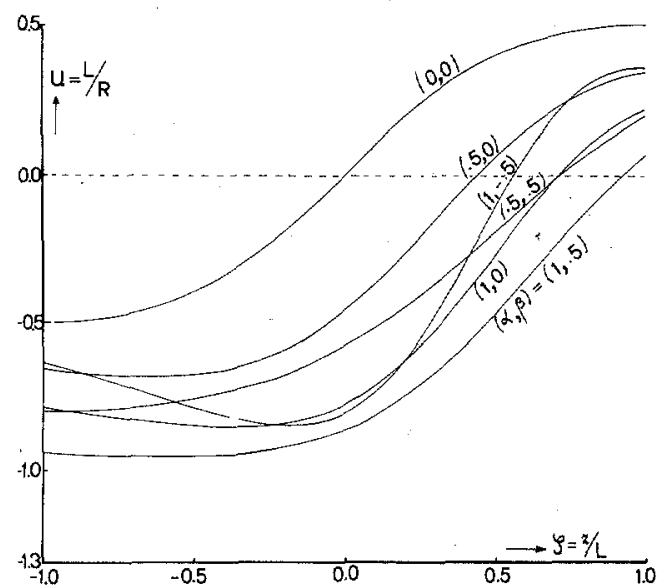

Fig.1. The curvature of the phase front along the cavity of a semiconfocal system in terms of $\zeta=z / L$, where $L$ is the distance between the mirrors. The flat mirror is at $\zeta=0$ and the curved mirror is at $\zeta= \pm 1$. The wave propagation from the flat mirror is plotted for $\zeta>0$ and the other one for $\zeta<0$. The values of the parameters $\alpha$ and $\beta$ are indicated.

Using (2) and (9) we get:

$$
\frac{1}{E} \frac{d E}{d \zeta}=-j\left(\beta_{0} L-Q\right)+\left(\alpha_{0} L-U\right) .
$$

From this equation the amplitude and phase of the electric field strength can be obtained. It is clear that both amplitude and phase depend only on the gain —and index of refraction-values at the axis of the tube. The radial dependence is fully accounted for by $Q(\zeta, \beta, \alpha)$ and $U(\zeta, \beta$, $\alpha)$.

In the next section we further evaluate the gain factor $\alpha_{0}$ in terms of interaction of radiation intensity and active medium. For that purpose it is convenient to write (16) in terms of intensity $I$ at the optical axis instead of field strength. We then obtain:

$$
\frac{1}{I} \frac{d I}{d \zeta}=2\left(\alpha_{0} L-U\right)
$$

where $\alpha_{0} L$ is the amplitude gain for one passage through the medium of length $L$.

It is seen from (17) that the intensity of a propagating Gaussian beam is locally changed not only due to the local inversion density but also by convergence or divergence, respectively, of the wavefront. The latter contribution is described by the second term on the right-hand side of (17). For a semiconfocal system we calculated the values of $U$ along the laser tube for various values of $\alpha$ and $\beta$. The results are plotted in Fig. 1. The flat mirror is at the position $\zeta=0$. The wave running to the flat mirror is plotted for negative values of $\zeta$, and the wave running from the flat mirror has positive values of $\zeta$.

\section{The Rate Equations for Two GaUSSIAN BEAMS}

In this section we shall derive the rate equations for the radiation fields of two Gaussian modes interacting with a homogeneously broadened medium. In the evaluation of the relevant physical processes we still assume a medium with quadratic profile and we shall make use of the formalism derived in the preceeding section. The principal advantage of this formalism is that the interaction of radiation and medium need be considered only on the optical axis.

As a homogeneously broadened medium one can, for instance, consider molecular laser systems having a large life time of upper laser levels, small natural linewidth, but very fast cross relaxation among the available levels. Excited levels of different rotational and Doppler shifted frequency are collisionally coupled to the states interacting with the field. The strong influence of cross relaxation on the gain saturation allows efficient molecular systems with a Doppler profile to be treated as homogeneously broadened [8]. The intensity gain factors of the two modes are then

$$
2 \alpha_{01} L=\frac{g_{1} G_{0}}{1+g_{1} I_{1} / I_{0}+g_{2} I_{2} / I_{0}}
$$

and

$$
2 \alpha_{02} L=\frac{g_{2} G_{0}}{1+g_{1} I_{1} / I_{0}+g_{2} I_{2} / I_{0}}
$$

where $g_{1}$ and $g_{2}$ are the values of the line-shape function at the frequencies of the two fields with radiation intensities $I_{1}$ and $I_{2}$, respectively. These intensities are the sum of the intensities of waves running to the right and to the left. $G_{0}$ and $I_{0}$ are the small-signal gain for one passage through the medium and the saturation parameter, respectively, both at the central frequency. For homogeneously broadened media the saturation parameter is inversely proportional to the line-shape function, consistent with the requirement that the maximum available output is independent of frequency. Hence in (18) and (19) we substituted for the saturation parameters $I_{0} / g_{1}$ and $I_{0} / g_{2}$.

Considering the first mode, the radiation density can be obtained by substituting (18) into (17) and integrating this equation. The integrated part coming from $\alpha_{01}$ depends, as can be seen from (18), on the line-shape function. If the curvature effect in (17) was neglected, the integration would yield the well-known result of the radiation density maximum at the central frequency. Using this result for a resonator system having one perfect reflecting mirror and one outcoupling mirror, it is found that, as long as the reflectivity of the transmitting mirror is not too low, the sum of the intensities of the running parts of a mode is more or less constant [9].

For our problem we shall introduce the approximation that the sum of the intensities of the two running parts is constant. This assumption is valid if the cross section varies little and the outcoupling is low. Anyhow, this approximation has no effect on the mechanism of radial radiation transport.

We now integrate (17) for the first mode over a round trip through the active medium. If the intensity just after reflection of the outcoupling mirror is $I_{1}{ }^{\prime}$ and its value after a round trip is $I_{1}$ " we find: 


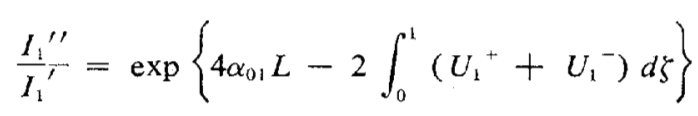

where $U_{1}^{+}$is equal to $L / R_{1}$ for the running wave to the right and similarly $U_{1}{ }^{-}$applies to the left running wave. It is seen from (20) that not the curvature of the wavefront itself counts, but the sum of the curvatures of the two running waves. In systems having no radial gain variation this curvature effect vanishes. The radiation intensity coupled out per unit area and unit time is equal to

$$
\frac{1-R}{1+R} I_{1}
$$

where $R$ is the reflectivity. The radiation gained per unit area and unit time is equal to

$$
\frac{R}{1+R} I_{1}\left[\exp \left\{4 \alpha_{01} L-2 \int_{0}^{1}\left(U_{1}^{+}+U_{1}^{-}\right) d \zeta\right\}-1\right]
$$

where it is assumed that the fractional change of $I_{1}$ (the sum of two running waves) is very small during the time of a round trip through the resonator or

$$
\frac{2 L}{c I d t} \ll 1
$$

where $c$ is the velocity of light.

The rate equation describing the radiation density is given by the difference between outcoupled radiation and radiation gained by the medium. We find

$$
\begin{aligned}
& \frac{1}{c} \frac{d I_{1}}{d t}=-\frac{1}{1} \frac{-}{+} \frac{R}{R} \frac{1}{L} I_{1}+\frac{R}{L(1+R)} I_{1} \\
& \quad\left[\exp \left\{4 \alpha_{01} L-2 \int_{0}^{1}\left(U_{i}^{+}+U_{1}^{-}\right) d \zeta\right\}-1\right] .
\end{aligned}
$$

Similarly, the rate equation describing the intensity $I_{2}$ of the second mode will be given by

$$
\begin{aligned}
\frac{1}{c} & \frac{d I_{2}}{d t}=-\frac{1}{1} \frac{-R}{1} \frac{1}{L} I_{2}+\frac{R}{L(1+R)} I_{2} \\
& \cdot\left[\exp \left\{4 \alpha_{02} L-2 \int_{0}^{1}\left(U_{2}^{+}+U_{2}^{-}\right) d \zeta\right\}-1\right] .
\end{aligned}
$$

\section{Time History of Gaussian Modes}

The problem is now to solve (21) and (22) in order to find the solutions for $I_{1}$ and $I_{2}$. Looking for an analytic time-dependent solution seems to be a difficult problem. However, since we are interested in the asymptotic values, it proves advantageous to study the values $I_{1}$ and $I_{2}$ graphically. We try to find the stationary solutions for $I_{1}$ and $I_{2}$ by following their temporal behavior in an $\left(I_{1}-I_{2}\right)$ plane. Through any point in the $\left(I_{1}-I_{2}\right)$ plane there is a trajectory that indicates the path followed by the respective points $\left(I_{1}, I_{2}\right)$ on their way to a stable state, with the time $t$ as parameter. See Fig. 2. The characteristics $L_{1}$ and $L_{2}$ of (21) and (22) are two families of curves in the $\left(I_{1}-I_{2}\right)$ plane, depending parametrically on $G_{0}$, because $G_{0}$ is in general a

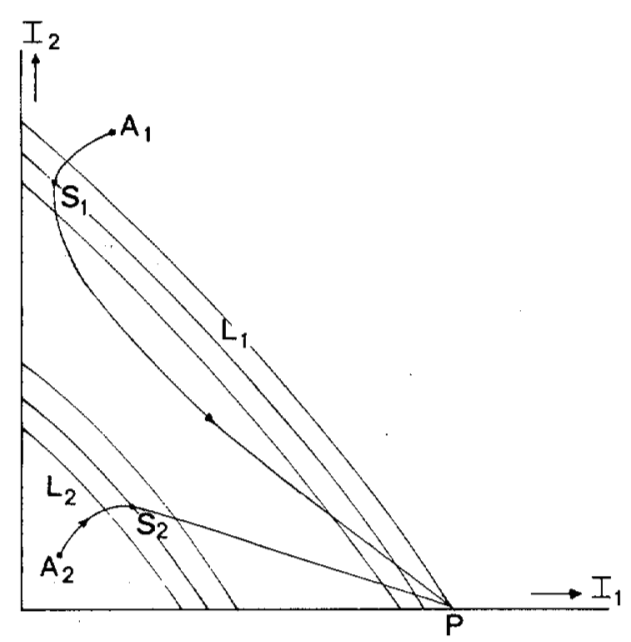

Fig. 2. Trajectories of unstable points in a $\left(I_{1}, I_{2}\right)$ diagram. Any pair of characteristics never cross each other. At the position where a trajectory crosses a characteristic the tangent is either horizontal or vertical depending on the characteristic. Mode competition leads to the survival of the mode with intensity $I_{1}$.

function of time, and obeying the equations

$$
\begin{aligned}
& L_{1}: \quad \alpha_{01} L=\frac{1}{2} \int_{0}^{1}\left(U_{1}{ }^{+}+{U_{1}}^{-}\right) d \zeta-\frac{1}{4} \ln R \\
& L_{2}: \quad \alpha_{02} L=\frac{1}{2} \int_{0}^{1}\left(U_{2}{ }^{+}+U_{2}{ }^{-}\right) d \zeta-\frac{1}{4} \ln R
\end{aligned}
$$

where $\alpha_{01}$ and $\alpha_{02}$, given by (18) and (19), respectively, are time-dependent. Since $g_{1}, g_{2}$, and $I_{0}$ are time-independent and the integrals are functions of $G_{0}(t), I_{1}$, and $I_{2}$, it is seen that all characteristics $L_{1}$ and $L_{2}$ can never cross each other.

If we divide (21) by (22) it is seen that any trajectory has a vertical tangent where it crosses $L_{1}$ and a horizontal tangent where it crosses $L_{2}$. Furthermore, if a point $\left(I_{1}, I_{2}\right)$ is located "below" or "above" the characteristic $L_{1}$, the corresponding trajectory indicates that as $t$ increases, $I_{1}$ increases or decreases, respectively. Similarly, if this point is located below or above $L_{2}, I_{2}$ will increase or decrease, respectively. These observations make it possible to derive the asymptotic values of $I_{1}$ and $I_{2}$ graphically.

Let us assume that the characteristic $L_{1}$ lies above $L_{2}$, as indicated in Fig. 2, for instance because the integral over $U$ in (23) is smaller than in (24) or because $g_{1}$ is larger than $g_{2}$. We follow a trajectory that starts at $A_{1}$ located above $L_{1}$ and $L_{2}$. Since $A_{1}$ lies above $L_{1}$ and $L_{2}$, the values of both $I_{1}$ and $I_{2}$ decrease. Subsequently, if the trajectory does not cross any of the characteristics, the integration reveals that both $I_{1}$ and $I_{2}$ become equal to zero. Let us now suppose that the trajectory crosses one of the characteristics. A nonzero stable value of $I_{1}$ will be found.

What happens if the trajectory has crossed, for instance, $L_{1}$ at the point $S_{1}$ and time $t_{0}$ ? We are especially interested in how the characteristic $L_{1}$ moves as the trajectory goes through $S_{1}$. At this point the derivative $d I_{2} / d I_{1}$ is infinite and the tangent to the trajectory is vertical. Suppose that at this point the characteristic moves with the trajectory, because they are both time-dependent, but so that the following points $\left(I_{1}, I_{2}\right)$ are still on the characteristics. The 
trajectory remains parallel to the $I_{2}$ axis and the final value of $I_{1}$ will then be given by the intersection of this line and the $I_{1}$ axis. This value of $I_{1}$ will in general not satisfy (21) and (22) as a steady-state solution. In other words, the characteristic will, in general, not move with the trajectory so that the point $\left(I_{1}, I_{2}\right)$ at the same time $t$ after $t_{0}$ will be located somewhere between $L_{1}$ and $L_{2}$. Between these two characteristics $I_{2}$ still decreases whereas $I_{1}$ increases. This means that the trajectory will never cross $L_{1}$ again, because at such an intersection the tangent to the trajectory becomes vertical implying that $I_{2}$ increases, which is prohibited by (22). Further, as the trajectory proceeds it will also never cross a characteristic $L_{2}$, because in crossing such a characteristic the tangent of the trajectory would have to become horizontal, implying that $I_{1}$ decreases, which is impossible. Since the characteristics $L_{1}$ and $L_{2}$ will never cross each other the trajectory will finally reach the point $P$ on the axis. At this stable point the values of the intensities are given by

$$
I_{2}=0
$$

$\frac{I_{1}}{I_{0}}=G_{0}\left\{\int_{0}^{1}\left(U_{1}^{+}+U_{1}^{-}\right) d \zeta-\frac{1}{2} \ln R\right\}^{-1}-g_{1}{ }^{-1}$.

Let us consider next the trajectory starting from $A_{2}$, located below $L_{1}$ and $L_{2}$, as indicated in Fig. 2. Initially, $I_{1}$ and $I_{2}$ will both increase until one of the characteristics, in this case $L_{2}$, is crossed at $S_{2}$. Analogous to what has been mentioned above the trajectory of unstable points passing through $S_{2}$ will never again cross any of the characteristics $L_{1}$ and $L_{2}$ on its way to a stable value. Because $L_{1}$ and $L_{2}$ never cross each other the trajectory must finally also reach point $P$ of the $I_{1}$ axis and the values of the two intensities are given again by (25) and (26).

Now let us assume that the characteristics $L_{2}$ lie above $L_{1}$ as indicated in Fig. 3. We find similarly that starting from the points $A_{3}$ and $A_{4}$ the stable point $Q$ on the $I_{2}$ axis will be reached. For that case the values of $I$ are given by

$$
I_{1}=0
$$

$\frac{I_{2}}{I_{0}}=G_{0}\left\{\int_{0}^{1}\left(U_{2}{ }^{+}+U_{2}^{-}\right) d \zeta-\frac{1}{2} \ln R\right\}^{-1}-g_{2}^{-1}$.

The numerical evaluation of the integrals given in (26) and (28) leads only to nonzero values if we are dealing with a medium having gain variations. In [5] we have shown that the radius of curvature of the wavefront at the mirror is only equal to that of the mirror if gain variations are absent. It can also be shown that for a medium with no gain variations at any place between the mirrors the value of the radius of curvature of the wavefront of a running wave to the left is equal to minus the value of the wave running to the right; i.e., the two wavefronts coincide. Consequently, the integral becomes zero and we conclude from (26) and (28) that the mode with the larger line-shape value $g$ will survive at the expense of the other one. In other words, if we have a symmetrical line shape, monotonically decreasing from the center, the mode with frequency closest to the central frequency will oscillate.

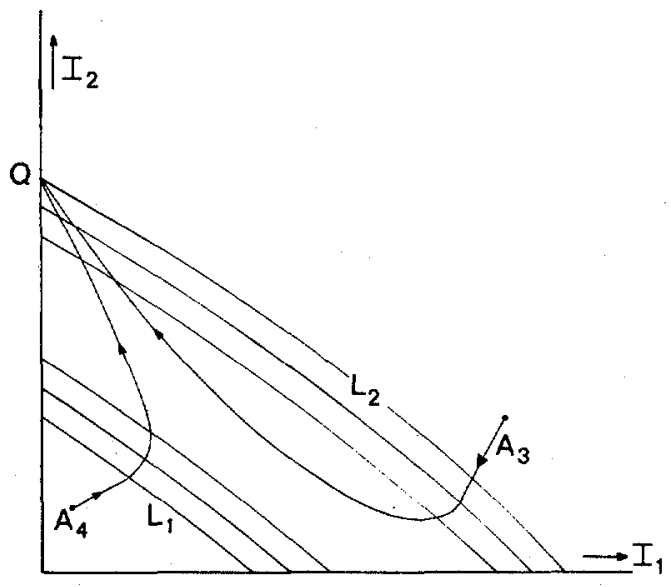

Fig. 3. Trajectories of unstable points in a $\left(I_{1}, I_{2}\right)$ diagram. In this case, mode competition leads to the survival of the mode with intensity $I_{2}$.

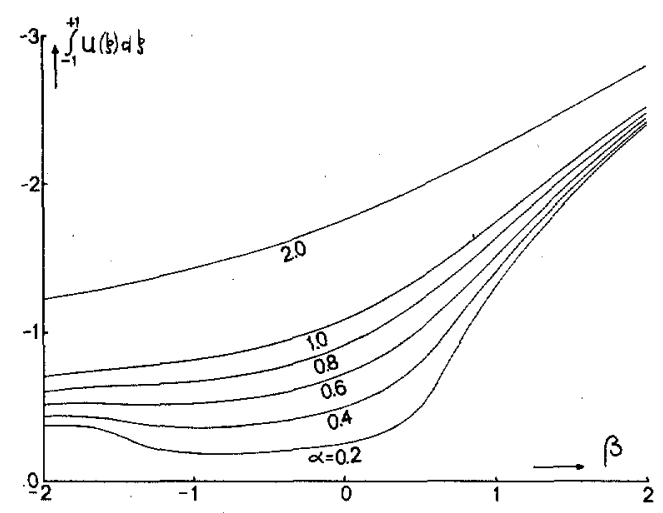

Fig. 4. The curvature of the phase front integrated for one loop through the active medium as a function of $\beta$ for different values of $\alpha$. The calculations are made for the semiconfocal system.

However, in the case of gain variations the two wavefronts do not coincide and it turns out that the integral of (26) and (28) is nonzero. Its value depends on mirror configuration, $\alpha$ and $\beta$. In that case, the surviving mode will not necessarily be the one with frequency closest to the line center. This will be further analyzed in Section VI. In order to have an idea about the values of the integrals in (26) and (28) we have plotted their numerical values as a function of $\beta$ with $\alpha$ as a parameter. It is done in Fig. 4 for a semiconfocal system. An analytic expression of the integral is given in the Appendix.

The present theory of competition between two Gaussian modes in a medium with homogeneous line broadening can be easily extended to the case of competition between more than two Gaussian modes. In the case of $n$ modes we have to follow trajectories in $n$-dimensional space and the characteristics are $(n-1)$-dimensional "planes." The analysis is similar to what has been described. In the steady state only one mode oscillates for which the final intensity is maximum.

\section{Description of Gain Profile}

As we mentioned, the presence of a gain profile is essential for finding a nonzero value for a round-trip integral of the curvature of the wavefront. In this section, we shall discuss gain variations in laser systems and we shall look 
for the main quadratic term near the axis where the beam intensity is highest. In the present treatment, dealing with homogeneous line broadening, the amplitude gain at a distance $r$ from the axis is simply given by

$$
\delta_{r}=\frac{\frac{1}{2} G_{r} / L}{1+I_{r} / I_{s r}}
$$

where $G_{r}$ is the small signal intensity gain for one passage, $I_{r}$ is the intensity of the beam, and $I_{s r}$ is the saturation parameter. For systems having axial symmetry we use a serial expansion in terms of the distance to the axis. Thus

$$
\delta_{r}=\alpha_{0}+\frac{1}{2} \alpha_{2} r^{2}+\cdots .
$$

Next we substitute into (29) the following expressions for $G_{r}, I_{r}$, and $I_{s r}$ :

$$
\begin{aligned}
& G_{r}=G\left(1+\frac{1}{2} \gamma r^{2}\right) \\
& I_{s r}=I_{s}\left(1+\frac{1}{2} \epsilon r^{2}\right) \\
& I_{r}=I \exp -2 r^{2} / \omega^{2} .
\end{aligned}
$$

Comparing the result with (30) we find

$$
\alpha_{2}=\frac{\frac{1}{2} G / L}{1+I / I_{s}}\left\{\gamma+\frac{1}{1+I / I_{s}}\left(\epsilon+\frac{4}{\omega^{2}}\right) \frac{I}{I_{s}}\right\}
$$

For homogeneous line broadening $I_{s} G$ does not depend on frequency. This is a direct consequence of the fact that the maximum obtainable output in the case of homogeneous line broadening does not depend on the oscillating frequency. Thus we have $G=G_{0} \cdot g$ and $I_{s}=I_{0} / g$.

For the further numerical evaluation we assume that the line shape can be assumed as mainly Doppler broadened, which is reasonable for molecular systems with pressures up to about 5 torr. We get

$$
\alpha_{2}=\frac{\frac{1}{2} G_{0} / L}{\exp \left(\xi^{2}\right)+I / I_{0}}\left\{\gamma+\frac{\left(\epsilon+4 / \omega^{2}\right) I}{I+I_{0} \exp \left(\xi^{2}\right)}\right\}
$$

where $G_{0}$ and $I_{0}$ are, respectively, the small-signal gain and saturation parameter at the central frequency, and $\xi$ is a normalized frequency,

$$
\xi=2 \sqrt{\ln 2}\left(\nu-\nu_{0}\right) / \Delta \nu_{1}
$$

with $\nu_{0}$ the central frequency and $\Delta \nu_{D}$ the Doppler linewidth. Using the dimensionless form for the gain variation we obtain

$$
\left.\alpha=\frac{G_{0} I_{0}}{I_{0}} \underset{\exp \left(\xi^{2}\right)+I}{\xi^{2}+I \lambda L}+\frac{(\epsilon \lambda L / 4 \pi+Q) I}{I+I_{0} \exp \left(\xi^{2}\right)}\right\} .
$$

It is seen that $\alpha$ decreases with increasing intensity, except just above threshold.

\section{The Effect of the Dispersive Behavior of the Active Medium on Mode Selection}

In Section IV we have seen that the oscillating mode will be the one that reaches the highest intensity in the medium. In order to find this mode we have to look for the frequency for which the right-hand side of (26) is maximum. We assume at this point that there are very many resonance frequencies for axial modes with very small frequency spacing.

Evaluating the integral over the curvature we have to insert the line-shape function $g$. As discussed in the previous section we choose a Doppler profile.

From the Kramers-Kronig relations the variation $\Delta n$ in the refractive index at a frequency $\nu$ in the vicinity of $\nu_{0}$ is given by

$$
\Delta n=\frac{c}{\pi^{2} \nu} \int_{0}^{\infty} \frac{\nu^{\prime} \alpha_{0}^{\prime} d \nu^{\prime}}{\nu^{\prime 2}-\nu^{2}}
$$

where $2 \alpha_{0}{ }^{\prime}$ is the gain factor at frequency $\nu^{\prime}$ defined in such a way that the intensity of a plane wave traversing the medium increases with distance $z$ as $\exp \left(2 \alpha_{0}{ }^{\prime} z\right)$. In the limits of $\Delta \nu_{D} \ll<\nu_{0}$ and $\nu-\nu_{0} \ll<\nu_{0}$ and neglecting hole burning effects in homogeneous line broadening, (35) can be approximated by

$$
\Delta n=\frac{c \alpha_{0}}{\pi \nu_{0} \sqrt{\pi}} \int_{0}^{\xi} \exp \left(x^{2}\right) d x
$$

where $2 \alpha_{0}$ is the gain factor at the frequency $\nu$, for which $\Delta n$ is calculated. It should be noted that if we substitute in (36), $\alpha_{0}=\alpha_{c} \exp \left(-\xi^{2}\right)$, where $2 \alpha_{c}$ is the gain factor at the central frequency, we obtain the usual anomalous dispersion formula associated with a gain profile.

We have assumed a quadratic profile which can be described by $\frac{1}{2} \alpha_{2} r^{2}$. The associated quadratic refractive index profile can be similarly described by $\frac{1}{2} \Delta n_{d} r^{2}$ where $\Delta n_{a}$ according to (36) is given by

$$
\Delta n_{d}=\frac{c \alpha_{2}}{2 \pi \nu_{0} \sqrt{\pi}} \int_{0}^{\xi} \exp \left(x^{2}\right) d x .
$$

Using for the dispersion the dimensionless form defined by

$$
\beta_{d}=\Delta n_{d} L^{2}
$$

we find

$$
\beta_{d}=\frac{\alpha}{\sqrt{\pi}} \int_{0}^{\xi} \exp \left(x^{2}\right) d x .
$$

It is seen that the quadratic profile due to dispersion disappears for oscillation at the central frequency. Apart from the dispersion of the medium we also have to account for possible thermal effects to the index profile [6]. So we get

$$
\beta=\beta_{d}+\beta_{t}
$$

Knowing $\alpha$ and $\beta$ as function of $\xi$ given by (34), (39), and (40), the integral of the curvature can be obtained as a 


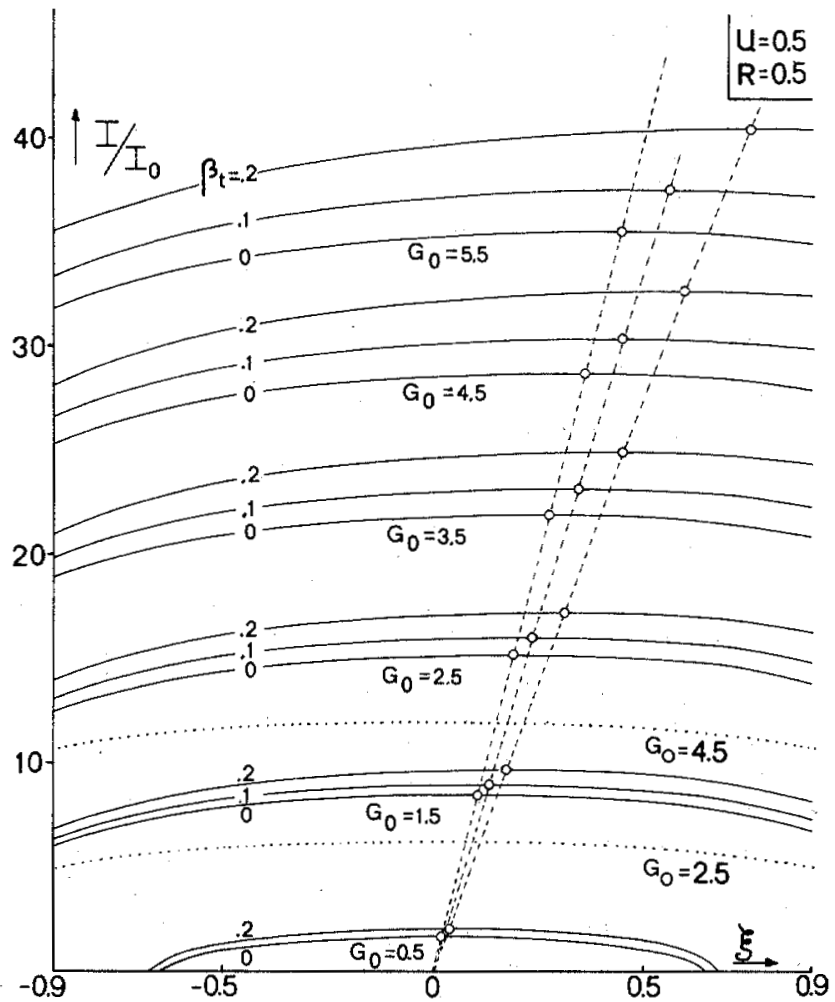

Fig. 5. Intensity characteristics are calculated for various values of $\beta$ and of the small-signal gain $G_{0}$. It is done for a semiconfocal system having a totally reflecting curved mirror and a flat outcoupling mirror with 50 -percent reflection. The characteristics are plotted as a function of the normalized frequency. For each condition given by $G_{0}$ and $\beta$, maximum intensity can be calculated. The corresponding frequencies are indicated by the intersections with the broken lines. For comparison two intensity characteristics have been drawn for the case that the radial radiation transport is not included (dotted lines).

function of frequency. Substituting the integral value into (26) the intensity $I / I_{0}$ as a function of frequency can be obtained. The results are plotted in Fig. 5 for the semiconfocal system. For the gain variation we have assumed that the main contribution comes from the intensity profile. Therefore, we have substituted $\gamma=\epsilon=0$ and for $Q$ the value at the flat mirror having a reflectivity of 50 percent. The intensities are given for different values of $G_{0}$ and $\beta_{t}$. It is seen that the frequency for which maximum intensity is obtained increases with the small-signal gain $G_{0}$, but also with increasing thermal effects. These maxima are the intersections with the broken lines indicated with the parameter $\beta_{t}$.

\section{Discussion}

We have seen that for long active media or high-power systems the curvature effect leads to a more complicated model of physical processes for finding laser characteristics than in the case of neglecting this effect. The numerical calculations have shown that the curvature of the phase front causes considerable radiation transport in the radial direction. This has far-reaching consequences for the gain and output characteristics. The new results deviate considerably from what has been predicted previously [10].

In the case of high-power systems where $I \gg I_{0}$ it is seen from (34) that $\alpha$ is roughly the saturated gain times $Q$.
Normally, the $Q$-value in a laser system varies between 0.5 and 1 , so that $\alpha$ can be estimated by knowing the saturated gain for passing once through the system. In general, one finds that the fractional increase of radiation at the axis of the system due to the radial transport is more or less comparable to the saturated gain. This is demonstrated in Fig. 5 , where for reasons of comparison the density ratio $I / I_{0}$ is also calculated for the case that the curvature effect was not included. It is done for $G_{0}=2.5$ and 4.5. In comparison it is seen that the inclusion of the curvature effect leads to an intensity change of more than a factor of 2 . The corresponding output density is then increased by the same ratio. Apart from the curvature effect, the gain variations and the thermal refractive index also change the beamwidth. In most practical situations there is an increase in beamwidth. This means that the output power of a single lowest order mode is considerably higher than what is predicted by a theory neglecting gain and index variations. Further, this radial radiation transport must also have a large effect on saturation parameter measurements [4]. This is an important reason why in experiments with narrow Gaussian beam (large gain variation) a larger saturation parameter is found if curvature effects are omitted than in experiments with a wider beam. Also in [11], where the normalized output is calculated for the simplest case of a plane-parallel resonator with a quadratic gain profile, the result would be very different if the curvature effect was included.

In the case of positive gain profile, i.e., increasing with the distance from the axis, the radial radiation transport increases with increasing frequency due to dispersion effects. If losses by spontaneous decay of the upper laser level were not taken into account, the oscillation frequency and also the inversion density would increase unlimitedly. The mechanism that restricts the frequency shift comes from the spontaneous emission competing with stimulating emission. One might expect that increasing reflectivity would decrease the frequency shift, because $\alpha$ and thus the curvature effect decreases with increasing $I$. However, the spontaneous loss also decreases with $I$. It turns out that the frequency shift increases slowly with $R$ and tends to a finite value for $R$ going to 1 . The surprising result is found that the more the medium is saturated, the more the frequency shifts from the line center.

It should be noted that the large influence of gain on dispersion is the main reason for such considerable frequency shift. We have seen that the dispersion is larger by a factor $\exp \left(\xi^{2}\right)$ than what is usually given for an inverted medium. The physical reason for this enlarged dispersion effect has to do with an increasing inversion density with increasing frequency shift from the line center. The inversion must increase because in the steady state of a laser system the total radiation gain and losses are balanced.

In the previous section, we looked for the frequency of maximum intensity. In practice, this frequency does not necessarily coincide with one of the resonance frequencies of the cavity. Therefore, we predict that the oscillation frequency will be a resonance frequency close to the frequency for which the maximum intensity was calculated. 


\section{APPENDIX}

The integration of the curvature of the phase front over one loop through the laser medium can be obtained as follows. From [5, eq. (10)] we find the complex beam parameter $P_{F}$ at the flat mirror by substituting $U_{1}=0$ :

$$
P_{k^{\prime}}= \pm\left[\frac{-U \cos ^{2} \eta-\eta \sin \eta \cos \eta}{\frac{1}{\eta} \sin \eta\left(\cos \eta-\frac{U}{\eta} \sin \eta\right)}\right]^{1 / 2}
$$

The sign choice depends on the condition that the imaginary part of $P_{F}$ has to be negative, so that $Q$ is positive.

Applying the matrix multiplication law we find the beam parameter at $z$ along the tube. Using $\zeta=z / L$ we obtain:

$$
P(\zeta)=\frac{-\eta \sin \eta \zeta+P_{F} \cos \eta \zeta}{\cos \eta \zeta+\frac{1}{\eta} \cdot P_{F} \sin \eta \zeta}
$$

The sum of the beam parameters of the two running waves reduces to a simple expression:

$$
P(\zeta)+P(-\zeta)=\frac{2 P_{F}}{\cos ^{2} \eta \zeta-\frac{\sin ^{2} \eta \zeta}{\eta^{2}} P_{F}{ }^{2}} .
$$

The integral (int) of the curvature of the phase front over one loop is the real part of the integral of (A2). Thus

$$
\text { int }=\operatorname{Re} \int_{0}^{i} \frac{2 P_{F} d \zeta}{\cos ^{2} \eta \zeta-\frac{\sin ^{2}}{\eta} \frac{\eta \zeta}{\eta} P_{F}{ }^{2}}
$$

The evaluation of the integral is straightforward; we find

$$
\begin{aligned}
\text { int }= & \pm \operatorname{Re} \log \left[\cos 2 \eta-\frac{U}{\eta} \sin 2 \eta\right. \\
& \left.-i\left\{\frac{U}{\eta} \sin 4 \eta+\left(1-\frac{U^{2}}{\eta^{2}}\right) \sin ^{2} 2 \eta\right\}^{1 / 2}\right]
\end{aligned}
$$

It should be remembered that $\eta$ is a complex function of $\alpha$ and $\beta$ given by (11).

\section{REFERENCES}

[1] W. E. Lamb, Jr, "Theory of optical maser," Phys. Rev., vol. 134, pp. A1429-A1450, June 1964.

[2] R. L. Fork and M. A. Pollack, "Mode competition and collision effects in gaseous optical masers," Phys, Rev., vol. 139, pp. A1408-A1414, Aug. 1965.

[3] W. J. Witteman, "Mode competition in lasers with homogeneous line broadening," IEEE J. Quantum Electron, vol. QE-5, pp. 92-97, Feb. 1969.

[4] C. P. Christensen, C. Freed, and H. A. Haus, "Gain saturation and diffusion in $\mathrm{CO}_{2}$ lasers," IEEE J. Quantum Electron, vol. QE-5, pp. 276-283, June 1969.

[5] W. J. Witteman, "High-power single-mode $\mathrm{CO}_{2}$ lasers," IEEE J. Quantum Electron (Special Issue on 1968 International Quantum Electronics Conference: Part II), vol, QE-4, pp. 786-788, Nov. 1968.

[6] G. J. Ernst and W. J. Witteman, "Mode structures of active resonators," IEEE J. Quantum Electron, vol. QE-9, pp. 911-918, Sept. 1973.

[7] H. Kogelnik, "On the propagation of Gaussian beams of light through lenslike media including those with loss or gain variation," Appl. Opt., vol. 4, pp. 1562-1599, Dec. 1965.

[8] A. Y. Cabezas and R. P. Treat, "Effect of spectral hole burning and cross relaxation on the gain saturation of laser amplifiers," J. Appl. Phys., vol. 37, pp. 3556-3563, Aug. 1966.

[9] A. Maitland and M. H. Dunn, Laser Physics. Amsterdam, The Netherlands: North-Holland Publishing Co., 1969, p. 186.

[10] W. W. Rigrod, "Saturation effects in high gain lasers," J. Appl. Phys., vol. 36, pp. 2487-2490, Aug. 1965.

[11] L. W. Casperson and A. Yariv, "Gain and dispersion focusing in a high gain laser," Appl. Opt., vol. 11, pp. 462-466, Feb 1972.

\title{
Generation of a Multigigawatt Nanosecond $\mathrm{CO}_{2}$ Pulse
}

\author{
YU-LI PAN, JOE R. SIMPSON, \\ ANTHONY F. BERNHARDT, AND STEPHEN E. KIERGAN
}

\begin{abstract}
A double-discharge TEA $\mathrm{CO}_{2}$ mode-locked oscillator/ amplifier system, which produces a maximum energy output of approximately $10 \mathrm{~J}$ in a single nanosecond pulse, is described. The modelocked oscillator operates on at least four lines simultaneously. The characteristics of the amplifying medium are also discussed.
\end{abstract}

Manuscript received June 18,1973 . This work was performed under the auspices of the U. S. Atomic Energy Commission.

The authors are with the Lawrence Livermore Laboratory, University of California, Livermore, Calif. 94550.

\section{INTRODUCTION}

I N. DESIGNING a laser system to obtain high-power nanosecond $\mathrm{CO}_{2}$ pulses for fusion research, it is advantageous to use components that are reliable, reproducible, and fully characterized. Some of the laser parameters of interest are small-signal gain, spatial-gain profiles, spectral content, and medium homogeneity. The construction and operation of a reliable and reproducible double-discharge TEA $\mathrm{CO}_{2}$ laser at 5-cm electrode separa- 